\title{
Antitumor Response of VEGFR2- and VEGFR3-Amplified Angiosarcoma to Pazopanib
}

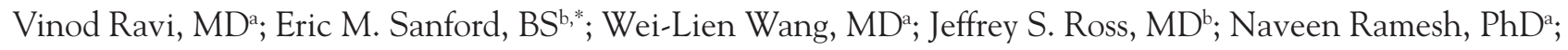 \\ Andrew Futreal, MDª Shreyaskumar Patel, MDa; Phillip J. Stephens, $\mathrm{PhD}^{\mathrm{b}}$; Vincent A. Miller, $\mathrm{MD}^{\mathrm{b}}$; and
}

Siraj M. Ali, MD, PhD

\begin{abstract}
Background: Angiosarcoma is a malignant neoplastic disease originating from or differentiating toward vascular endothelium, for which systemic pharmacologic treatment has limited durability. The molecular oncogenesis of angiosarcoma is often linked to inappropriate activations of vascular endothelial growth factor receptor (VEGFR) family members, which presents an opportunity for the use of therapy that selectively targets the machinery of vascular signaling. Methods: Hybridization capture of 3,320 exons of 182 cancer-related genes and the introns of 14 genes frequently rearranged in cancer was applied to more than 50 ng of DNA extracted from a formalin-fixed, paraffin-embedded biopsy of recurrent angiosarcoma and was sequenced to high, uniform coverage of 939x. Results: The angiosarcoma harbored amplifications of VEGFR2 (KDR) of 8 copies and VEGFR3 (FLT4) of 16 copies. The patient was initially treated with sorafenib, an inhibitor of VEGFR2, and developed progressive disease. The patient then received pazopanib, an inhibitor of VEGFR2 and VEGFR3 and experienced a potent antitumor response resulting in clinically stable disease for 6 months. Conclusions: This exceptional response to pazopanib treatment suggests that a subset of patients with angiosarcoma with genomic alterations in vascular signaling genes may respond well to pazopanib.
\end{abstract}

J Natl Compr Canc Netw 2016;14(5):499-502

\section{Background}

Metastatic or relapsed angiosarcoma initially responds well to cytotoxic chemotherapy, but the responses lack durability as the median time to progression is 4 to 5 months, indicating an acute need for more efficacious therapies. ${ }^{1,2}$ Because the oncogenesis of angiosarcoma is linked to the perturbation of vascular signaling, vascular endothelial growth factor receptor (VEGFR)-targeted therapy holds promise for effective treatment. ${ }^{3}$ However, clinical trials for patients with angiosarcoma have demonstrated limited activity with bevacizumab and sorafenib monotherapies, with response rates of $9 \%$ to $14 \% .4$ This report presents a patient with a sporadic,

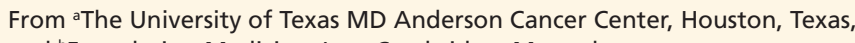
and ${ }^{b}$ Foundation Medicine, Inc., Cambridge, Massachusetts.

*Current affiliation: Perelman School of Medicine, University of Pennsylvania, Philadelphia, Pennsylvania.

Submitted April 4, 2014; accepted for publication February 10, 2016.

Dr. Ravi had disclosed that he has received research funding from GlaxoSmithKline. Dr. Patel has disclosed that he is a consultant for Johnson \& Johnson, CytRx Corporation, EMD Serono, Inc., Eli Lilly and cutaneous angiosarcoma harboring high-level amplifications of members of the VEGFR family, specifically VEGFR2 (KDR; 8x) and VEGFR3 (FLT4; 16x). In response to pazopanib, an inhibitor of both VEGFR2 and VEGFR3, the patient experienced disease control for 6 months, with early regression of clinically evident lesions within 4 weeks of commencing therapy.

\section{Case Report}

An 83-year-old man presented to his physician with an irregular erythematous patch on the right anterior scalp in February 2008. Surgical resection of the area and

Company, and Bayer. Mr. Sanford and Drs. Ross, Stephens, Miller, and Ali had disclosed that they are employees of and have equity interest in Foundation Medicine, Inc. The remaining authors have disclosed that they have no financial interests, arrangements, affiliations, or commercial interests with the manufacturers of any products discussed in this article or their competitors.

Correspondence: Vinod Ravi, MD, Department of Sarcoma Medical Oncology, The University of Texas MD Anderson Cancer Center, 1515 Holcombe Boulevard, Houston, TX 77030. E-mail: vravi@mdanderson.org 
pathologic examination of the specimen revealed a malignant neoplastic proliferation of blood vessels with irregular channels and atypical endothelial cells, consistent with a diagnosis of angiosarcoma. He presented to MD Anderson Cancer Center 3 weeks later for consultation at the sarcoma center, and underwent definitive hyperfractionated radiotherapy of 200 cGy per fraction times 30 fractions (total dose of 6,000 Gy), and the patient remained disease-free for 39 months.

In September 2011 the patient experienced facial swelling of the right cheek and visited his primary care physician. A punch biopsy of the cheek was performed, and subsequent histologic examination demonstrated recurrent angiosarcoma (Figure 1) outside the previous radiation field consistent with the multifocal nature of the disease in the head and neck region. A multidisciplinary tumor board at our institution recommended medical management because additional radiotherapy was contraindicated due to previous radiotherapy and the wide extent of recurrent disease. The patient was initially treated with paclitaxel at $80 \mathrm{mg} / \mathrm{m}^{2}$ weekly; however, he was unable to tolerate paclitaxel weekly without a treatment break. He was therefore maintained on paclitaxel at the $80 \mathrm{mg} / \mathrm{m}^{2}$ dose, cycling 2 weeks on and 1 week off for 9 months after initiating therapy in January 2012. After several months of treatment, the patient required a treatment holiday secondary to neurotoxicity in September 2012; progressive disease was noted shortly thereafter.

At this time, formalin-fixed, paraffin-embedded tissue from the recurrent angiosarcoma specimen from the right cheek was submitted to a CLIA-

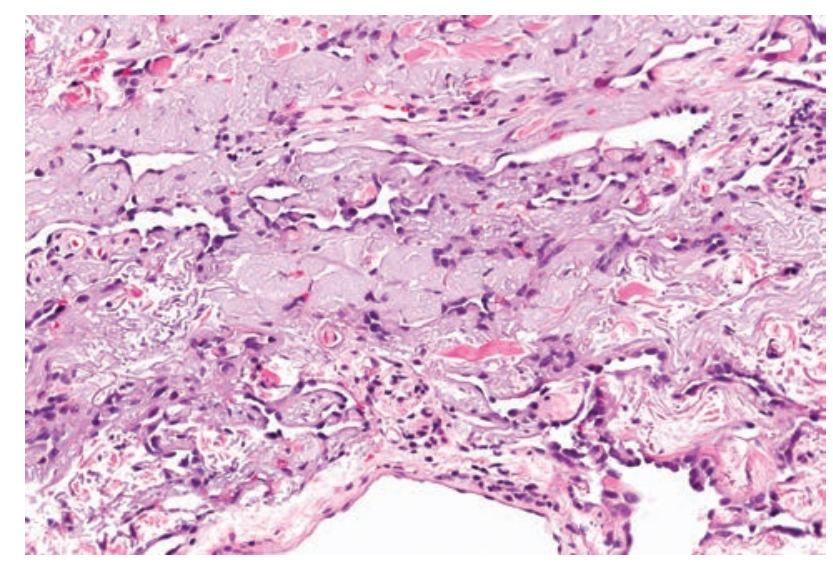

Figure 1. Right cheek skin punch biopsy reveals angiosarcoma. Tumor composed of infiltrative irregular vascular channels lined by atypical endothelial cells (hematoxylin-eosin, original magnification $\times 200$ ). certified, College of American Pathologists (CAP)accredited laboratory (Foundation Medicine, Inc.) for comprehensive genomic profiling using clinical next-generation sequencing. ${ }^{6}$ Hybridization capture of 3,320 exons of 182 cancer-related genes and 37 introns of 14 genes frequently rearranged in cancer was applied to more than $50 \mathrm{ng}$ of DNA extracted from this sample and sequenced to high, uniform coverage of 939x. All classes of cancer-related genomic alterations (including base substitutions, small insertions and deletions, rearrangements, and copy number alterations) were assayed for within the angiosarcoma specimen. The tumor was found to harbor 6 cancer-related somatic variants: VEGFR2 amplification (8x), VEGFR3 amplification (16x), MCL1 amplification (8x), TP53 R213*(2\%) and H179Y (4\%), and ARID1A Q2128* (14\%) in an estimated tumor background of $30 \%$ purity (Figure 2). VEGFR3 R1070L (67\%) was also observed, but its functional significance in cancer is currently unknown.

Because VEGFR2 base substitutions, but not amplifications, ${ }^{7}$ have been previously observed in angiosarcoma, we started the patient on a putative VEGFR inhibitor, sorafenib, at a dose of $400 \mathrm{mg}$ daily in November 2012, with the dose increasing to $800 \mathrm{mg}$ daily in December 2012. Despite observing $8 \mathrm{x}$ VEGFR2 amplification on comprehensive genomic profiling, this strategy was unsuccessful, as clinical disease progression was seen in a followup visit in February 2013, resulting in discontinuation of sorafenib. Because the patient had evidence of facial edema, a feature not typical for head and neck angiosarcomas, and because genomic profiling revealed amplification in VEGFR3, which plays a major role in lymphangiogenesis, a dual inhibitor of VEGFR2 and VEGFR3, pazopanib, was started in February 2013 at a dose of $400 \mathrm{mg}$ daily, and was subsequently increased to $800 \mathrm{mg}$ daily. The patient's right facial swelling was markedly reduced in 4 weeks (Figure 3), and high-resolution head and neck CT in April and June of 2013 further showed stable disease.

A physical examination conducted in June 2013 noted no progressive clinical disease, and a highresolution thoracic scan performed in July 2013 revealed no definitive evidence of metastatic disease. The patient was seen to have progressive disease in August 2013 and was switched to chemotherapy with gemcitabine and docetaxel. He showed a response to therapy after 2 cycles; however, he de- 


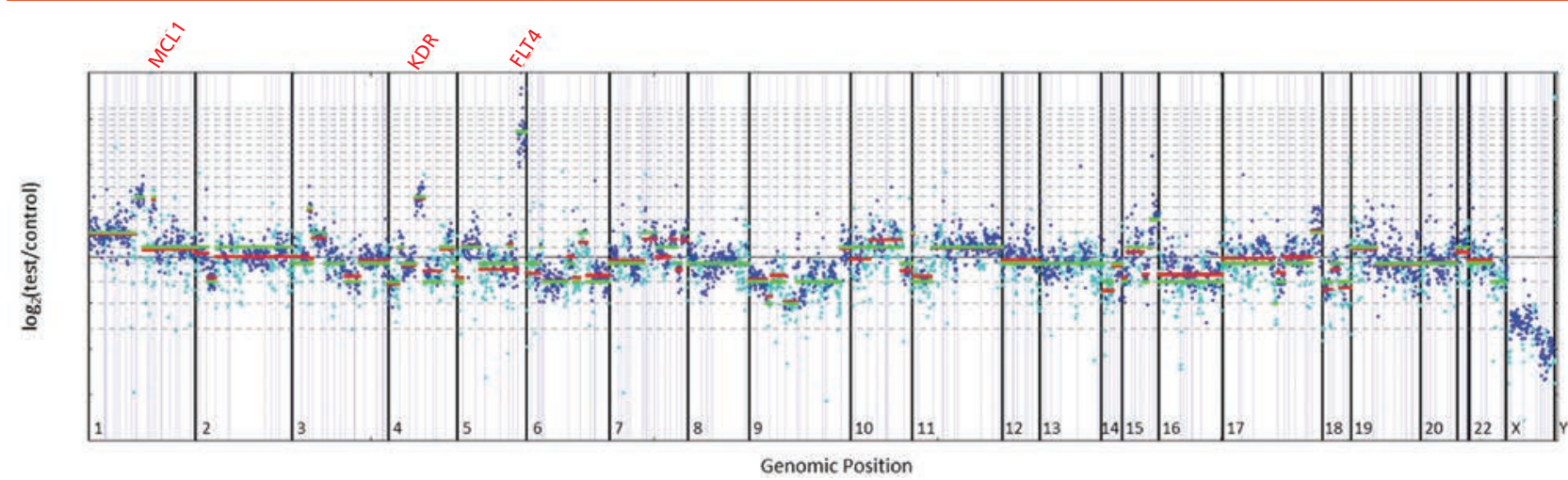

Figure 2. Genomic profiling identifies amplification of KDR (VEGFR2) and FLT4 (VEGFR3). Genomic profiling by a hybrid capture-based next-generation sequencing assay was performed on the resected specimen tissue to a coverage depth of 939x, revealing 6 distinct genomic alterations, including focal amplifications of KDR (8 copies) and FLT4 (16 copies). Copy number was determined by modeling copy variation and aneuploidy across the genome, and was compared with process-matched normal controls.

clined additional treatment due to fatigue that was disabling. He was then transitioned to hospice care. Pazopanib treatment was successful in producing a partial response that was sustained for 6 months. The durability of the response is impressive considering both the median progression-free survival (PFS) and the low response rate observed with sorafenib $(3.8$ months and $14 \%$, respectivley) ${ }^{5}$ and bevacizumab (3 months and 9\%, respectively) $)^{4}$ in angiosarcomas, and the median PFS observed with pazopanib (4.6 months) for sarcomas in general. ${ }^{8}$

\section{Discussion}

Angiosarcoma is a malignant neoplasm of vascularorigin derived from endothelial cells. ${ }^{9}$ Although comprising only $2 \%$ of soft tissue sarcomas, this dis-
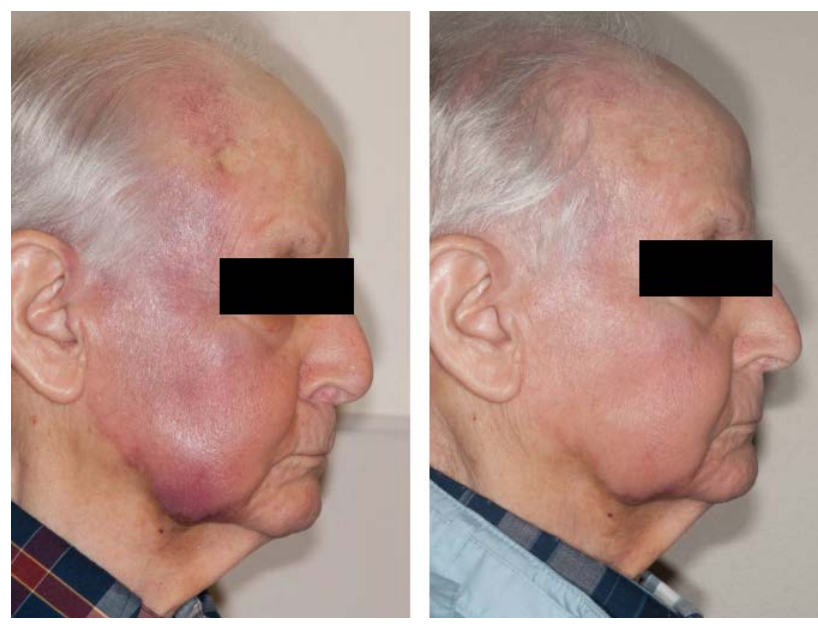

Figure 3. Extensive cutaneous angiosarcoma of the face affecting right parietal scalp, right temporal region, and right cheek. (A) Disease before initiation of pazopanib. (B) The same region of the face after 4 weeks of treatment with oral pazopanib at $800 \mathrm{mg}$ daily. ease carries a high mortality with a 5-year survival rate of only approximately $30 \% .{ }^{10}$ The disease often arises sporadically in the skin of the head and neck region or in anatomic sites secondary to specific insults, such as breast angiosarcoma after therapeutic radiation, soft tissue angiosarcoma induced by lymphedema, and liver angiosarcoma after exposure to vinyl chloride. ${ }^{10}$ Definitive diagnosis of angiosarcoma is made through histopathologic examination of tumor tissue, which shows ill-formed, anastomosing vessels with anaplastic, pleomorphic cells. The cells can be shown to be of endothelial origin by immunohistochemical staining for vascular markers such as CD31, CD34, and D2-40.

The NCCN Clinical Practice Guidelines in Oncology (NCCN Guidelines) for Soft Tissue Sarcoma recommend surgery and radiotherapy as the primary mode of treatment for local control of angiosarcoma, with incorporation of systemic therapy often being essential due to the high locoregional and distant recurrence risk in this disease. In the case of metastatic or unresectable disease, the use of cytotoxic agents and targeted therapies forms the mainstay of therapy. ${ }^{11}$ Of the recommended agents, pazopanib in particular presents an interesting therapeutic hypothesis, because it targets the underlying derangements in VEGFR signaling that are frequent in angiosarcoma.

Several lines of evidence suggest that the inappropriate activation of VEGFR signaling is critical in the genesis of angiosarcoma., ${ }^{3,910,12}$ Physiologically, VEGFR2 and VEGFR3 regulate angiogenesis and lymphangiogenesis, respectively. ${ }^{13,14}$ In most angiosarcoma cases, VEGFR1-3 are overexpressed as characterized by immunohistochemistry. ${ }^{13,14}$ More- 
over, activating base substitutions in VEGFR2 were observed in 4 of 39 patients with angiosarcoma, ${ }^{9}$ and VEGFR3 amplifications identified by fluorescence in situ hybridization using bacterial artificial chromosomes are present in 5 of 20 angiosarcoma cases secondary to radiotherapy. ${ }^{10}$

Pazopanib targets the VEGFR family, but has a low response rate of $6 \%$ in clinical trials for sarcoma. ${ }^{8}$ It is a tyrosine kinase inhibitor that was approved by the FDA for use in advanced soft tissue sarcoma based on the results of the phase III PALETTE trial. ${ }^{8}$ Although it is clear that pazopanib is not uniformly effective in all cases of sarcoma, identifying patients with increased likelihood of response has been a major challenge. Identification of genomic alterations may help further subclassify the heterogeneous group of tumors that share a common histopathologic diagnosis of angiosarcoma. Given the heterogeneity among angiosarcomas, both in terms of response to pazopanib and genomic alterations, this patient course highlights a possible correlation between responsiveness to pazopanib and amplification of VEGFR2 and VEGFR3.

Both sorafenib and pazopanib inhibit VEGFR family members among other targets. In one biochemical assessment of purified kinase activity cited in a regulatory filing, the apparent inhibition constant $\left(\mathrm{K}_{\mathrm{i}}^{\mathrm{app}}\right)$ for both inhibitors against VEGFR2 and VEGFR3 was in the single nanomolar range. ${ }^{15}$ However, another published study demonstrated a greater potency of pazopanib relative to sorafenib for VEGFR family members. ${ }^{16}$ The VEGFR2 IC50 for sorafenib was $150 \mathrm{nM}$, whereas IC50 for pazopanib was $51 \mathrm{nM}$ when kinase assays were performed at an ATP concentration of $1 \mathrm{mM}$, a level that mimics intracellular physiology. ${ }^{16}$ The VEGFR3 IC50 was 130 and $100 \mathrm{nM}$, respectively, for sorafenib and pazopanib. ${ }^{16}$ The relevance of inhibitory constants for biochemical kinase assays to in vivo effects is unclear, and is subject to the conditions of the assay, but the referenced findings hint at a possible explanation for the differing responses of this patient to pazopanib and sorafenib. Other possibilities include off-target effects of these inhibitors, but further work is needed to define a possible mechanism for this effect.

This patient's case may represent a subset of highly pazopanib-responsive angiosarcomas characterized by highly activating genomic alterations of VEGFR family members. Other investigators have described a response to pazopanib in a patient with cardiac angiosarcoma with regression of pericardial involvement and effusions, ${ }^{17}$ but they did not perform genomic profiling. Similarly, the genomic profiles of the $6 \%$ of patients with soft tissue sarcoma who experienced a partial response to pazopanib in the PALETTE trial are also unknown. Retrospective profiling of these patients' tumors may corroborate the observation that activating alterations, particularly high-level amplifications, of VEGFR family members predict superior outcomes with pazopanib. To further explore the possibility of a genomically defined pazopanib-responsive subset of patients with angiosarcoma, prospective clinical trials accompanied by comprehensive genomic profiling are needed.

\section{References}

1. Fata F, O'Reilly E, Ilson D, et al. Paclitaxel in the treatment of patients with angiosarcoma of the scalp or face. Cancer 1999;86:2034-2037.

2. Fury MG, Antonescu CR, Van Zee KJ, et al. A 14-year retrospective review of angiosarcoma: clinical characteristics, prognostic factors, and treatmen outcomes with surgery and chemotherapy. Cancer J 2005;11:241-247.

3. Park MS, Ravi V, Araujo DM. Inhibiting the VEGF-VEGFR pathway in angiosarcoma, epithelioid hemangioendothelioma, and hemangiopericytoma/ solitary fibrous tumor. Curr Opin Oncol 2010;22:351-355.

4. Agulnik M, Yarber JL, Okuno SH, et al. An open-label, multicenter, phase II study of bevacizumab for the treatment of angiosarcoma and epithelioid hemangioendotheliomas. Ann Oncol 2013;24:257-263.

5. Maki RG, D'Adamo DR, Keohan ML, et al. Phase II study of sorafenib in patients with metastatic or recurrent sarcomas. J Clin Oncol 2009;27:31333140 .

6. Frampton GM, Fichtenholtz A, Otto GA, et al. Development and validation of a clinical cancer genomic profiling test based on massively parallel DNA sequencing. Nat Biotechnol 2013;31:1023-1031.

7. Guo T, Agaram NP, Wong GC, et al. Sorafenib inhibits the imatinib-resistant KITT670I gatekeeper mutation in gastrointestinal stromal tumor. Clin Cancer Res 2007;13:4874-4881.

8. van der Graaf WT, Blay JY, Chawla SP, et al. Pazopanib for metastatic soft-tissue sarcoma (PALETTE): a randomised, double-blind, placebo-controlled phase 3 trial. Lancet 2012;379:1879-1886.

9. Antonescu CR, Yoshida A, Guo T, et al. KDR activating mutations in human angiosarcomas are sensitive to specific kinase inhibitors. Cancer Res 2009;69:7175-7179.

10. Guo T, Zhang L, Chang NE, etal. Consistent MYC and FLT4 gene amplification in radiation-induced angiosarcoma but not in other radiation-associated atypical vascular lesions. Genes Chromosomes Cancer 2011;50:25-33.

11. von Mehren M, Randall RL, Benjamin RS, et al. NCCN Clinical Practice Guidelines in Oncology; Soft Tissue Sarcoma. Version 2.2016. Accessed April 4,2016 . To view the most recent version of these guidelines, visit NCCN.org.

12. Ravi V, Patel S. Vascular sarcomas. Curr Oncol Rep 2013;15:347-355.

13. Jussila L, Alitalo K. Vascular growth factors and lymphangiogenesis. Physiol Rev 2002;82:673-700.

14. Yancopoulos GD, Davis $S$, Gale NW, et al. Vascular-specific growth factors and blood vessel formation. Nature 2000;407:242-248.

15. European Medicines Agency. CHMP assessment report: Votrient. Available at: http://www.ema.europa.eu/docs/en_GB/document_library/EPAR__Public_ assessment_report/human/001141/WC500094275.pdf, Accessed April 3, 2016

16. Kitagawa D, Yokota K, Gouda M, et al. Activity-based kinase profiling of approved tyrosine kinase inhibitors. Genes Cells 2013;18:110-122.

17. Ong P, Greulich S, Schumm J, et al. Favorable course of pericardial angiosarcoma under Paclitaxel followed by pazopanib treatment documented by cardiovascular magnetic resonance imaging. Circulation 2012;126:e279-281. 